\title{
Dinâmica sazonal de carrapatos (Acari: Ixodidae) na mata ciliar de uma área endêmica para febre maculosa na região de Campinas, São Paulo, Brasil
}

\author{
Seasonal dynamics of ticks (Acari:Ixodidae) in an endemic area for spotted fever in the Campinas region, \\ state of São Paulo, Brazil
}

\author{
Savina Silvana Aparecida Lacerra de Souza ${ }^{1}$ Celso Eduardo de Souza ${ }^{2}$ Elias José Rodrigues Neto ${ }^{3}$ \\ Angelo Pires do Prado ${ }^{3}$
}

\section{RESUMO}

A febre maculosa é mundialmente reconhecida como um problema reemergente de saúde pública. Na região de Campinas-SP, observam-se uma ampliação da área de transmissão do agente da doença e a ocorrência de um maior número de casos confirmados nos últimos anos. Nesta região, a maioria dos casos desta doença está quase sempre relacionada com o aumento populacional de capivaras, que são um dos principais hospedeiros primários do estádio adulto do carrapato Amblyomma cajennense. O principal objetivo deste estudo foi determinar o comportamento populacional de larvas, ninfas e adultos de Amblyomma spp no habitat de mata ciliar de uma área endêmica. De novembro de 2000 a outubro de 2002, carrapatos de vida livre foram coletados com armadilhas de $\mathrm{CO}_{2}$. Picos populacionais de larvas do gênero Amblyomma se estenderam por praticamente todos os meses do ano. Ninfas do gênero Amblyomma ocorreram o ano todo na mata ciliar, sendo mais abundantes de julho a dezembro. Adultos de A. cajennense foram mais abundantes na estação de primavera e verão. Adultos de A.dubitatum (=Amblyomma cooperi) apresentaram um padrão sazonal diferenciado, com os maiores picos populacionais ocorrendo de agosto a fevereiro.

Palavras-chave: Amblyomma cajennense, Amblyomma dubitatum, febre maculosa, mata ciliar.

\section{ABSTRACT}

Spotted fever is recognized worldwide as a reemerging public health problem. In Campinas region, state of São Paulo, the transmission area has expanded and the number of positive cases has increased in the lastest years. In these region most cases are usually related to the increased number of capybaras, which are one of the main primary hosts for the tick Amblyomma cajennense. The main objective of this study was to determine the seasonal dynamic of larvaes, nymphs and adults of Amblyomma species in a gallery forest. From November 2000 to October 2002 free-living ticks were collected using carbon dioxid traps. Larvae of Amblyomma spp were abundant during almost all months. Nymphs of Amblyomma spp occurred during the whole year and were more abundants from July to December. Adults of A.cajennense were more abundants during the spring-summer months. Adults of A.dubitatum showed higher populational peaks from August to February.

Key words: Amblyomma cajennense, Amblyomma dubitatum, spotted fever, gallery forest.

\section{INTRODUÇÃO}

A febre maculosa brasileira (FMB) é uma doença de caráter endêmico na região de CampinasS.P., com vários municípios apresentando casos humanos desde 1985. No município de Jaguariúna-SP, até $\mathrm{o}$ ano de 2000, ocorreram 15 casos da doença (LIMA et al., 2003). Um dos prováveis locais de infecção foi a Fazenda Santa Júlia, onde uma paciente residente naquela localidade referiu frequentar a mata ciliar, próxima à sua residência.

A FMB tem como agente etiológico a bactéria Rickettsia rickettsii Wollbach. Esta riquetsiose é transmitida por carrapatos da família Ixodidae, que são vetores e reservatórios (BURGDORFER, 1975; DIAS \& MARTINS, 1939). No Brasil, o mais importante

${ }^{1}$ Superintendência de Controle de Endemias (SUCEN-SR05), Campinas, Rua São Carlos, 546, Parque Itália, Fone/fax: (19)32726823/ 32729891, 13045-420, Campinas, SP, Brasil. E-mail: sr05@sucen.sp.gov.br. Autor para correspondência.

2SUCEN-SR05, Campinas, SP, Brasil.

${ }^{3}$ Departamento de Parasitologia, Instituto de Biologia, Universidade Estadual de Campinas (UNICAMP), Campinas, SP, Brasil. 
vetor é o carrapato de cavalo ou carrapato estrela, Amblyomma cajennense Fabricius (ARAGÃO \& FONSECA, 1961; MAGALHÃES, 1952; TRAVASSOS \& VALLEJO-FREIRE, 1944-1945). Os hospedeiros primários desta espécie são os eqüinos, antas e capivaras (ARAGÃO, 1936). Um destes animais deve estar presente em uma área para que uma população de carrapatos se estabeleça, pois estas espécies são essenciais para a multiplicação da fase adulta do A.cajennense, que se reproduz com sucesso em poucos mamíferos, como os citados. Além disso, o habitat é o elemento determinante para a abundância de carrapatos em uma área. Nesse sentido, a mata ciliar da região representa um ótimo habitat, pois além da presença de capivaras, a cobertura vegetal e a umidade podem suportar grandes populações de carrapatos.

Apesar de poucos estudos ecológicos terem sido realizados nesta região, vários pacientes com FMB referiram contato com o remanescente de mata ciliar para lazer; portanto, este estudo teve como objetivo conhecer a atividade sazonal de carrapatos Amblyomma spp neste habitat.

\section{MATERIAL E MÉTODOS}

Os ixodídeos de vida livre foram coletados na Faz. Santa Júlia, em Jaguariúna-SP (2244’08”S e 4657’09”W, altitude: 570m). O delineamento da pesquisa consistiu em coletar os carrapatos a cada quinze dias, no período de novembro de 2000 a outubro de 2002, do remanescente de mata ciliar. A técnica utilizada foi a armadilha atrativa de $\mathrm{CO}_{2}$, modificada da original empregada por WILSON et al.(1972). Em média, 800 gramas de cubos de gelo seco foram colocados, quinzenalmente, em três pontos distintos na mata ciliar, em uma depressão no solo (total de três armadilhas por quinzena). Acima desta, foi colocada uma flanela branca $(1,0 \mathrm{~m} \mathrm{X} \mathrm{1,0m),} \mathrm{com} \mathrm{fita} \mathrm{adesiva} \mathrm{dupla} \mathrm{face} \mathrm{de} 1 \mathrm{~cm}$, de largura, fixada nas bordas e nos quatro lados da flanela. O tempo de permanência de cada armadilha foi de uma hora. As flanelas das armadilhas foram acondicionadas individualmente em sacos plásticos e encaminhadas ao laboratório, onde foram deixadas por um período de 10 minutos no “freezer”, a $-20^{\circ} \mathrm{C}$, para posterior identificação e contagem dos espécimes. Os carrapatos foram preservados em álcool 70\% e, para a identificação taxonômica das espécies, fez-se uso de microscópio estereoscópico com iluminação incidente e chaves dicotômicas descritas por ROBINSON (1926), ARAGÃO \& FONSECA (1961), JONES et al. (1972), GUIMARÃES et al. (2001). As larvas e ninfas foram identificadas ao nível de gênero, de acordo com as características dos adultos. Os dados meteorológicos relativos às temperaturas (mínima, média e máxima), à umidade relativa e à precipitação pluviométrica foram obtidos do Centro de Pesquisas Meteorológicas e Climáticas Aplicadas à Agricultura (CEPAGRI/ UNICAMP). Os gráficos de distribuição sazonal foram elaborados no programa Microsoft EXCEL ${ }^{\circledR}$. O “n” dos gráficos representa a soma do número médio de carrapatos por espécie e/ou estádio coletados na primeira e segunda quinzena aplicado à função logarítimica $(\log [n+1])$.

\section{RESULTADOS}

Os dados climáticos da região estão representados na figura 1 . De abril a outubro, o clima foi frio e seco, e, de novembro a março, quente e chuvoso. De acordo com a figura 2, observou-se alta infestação de larvas do gênero Amblyomma na mata ciliar na maioria dos meses do estudo. As ninfas, também do gênero Amblyomma, ocorreram em todos os meses do ano, sendo mais abundantes de julho a dezembro (Figura 3). A identificação da espécie de larvas e ninfas de Amblyomma spp não foi possível devido à inexistência de chaves taxonômicas para tal identificação específica. O aumento populacional do estádio adulto (machos e fêmeas) de A. cajennense ocorreu na estação mais quente e chuvosa do ano, de outubro a abril (Figura 4). A distribuição populacional de adultos de A.dubitatum Neumann (= A. cooperi) (machos e fêmeas) mostrou um aumento populacional a partir de agosto e declínio a partir de março (Figura 5).

\section{DISCUSSÃO}

As armadilhas de $\mathrm{CO}_{2}$ foram eficientes para a coleta de todos os estádios de Amblyomma spp, corroborando com SOLBERG et al.(1992) e WILSON et al. (1972), que referiram serem estas armadilhas eficientes para a coleta de todos os estádios de $\boldsymbol{A}$. americanum, nos Estados Unidos.

Neste estudo, observamos que, na mata ciliar, os picos de larvas de Amblyomma spp ocorreram praticamente o ano todo, diferindo de outros trabalhos em áreas de campo, com a presença apenas de A.cajennense, na Argentina (GUGLIELMONE \& HADANI, 1982), Trinidad (SMITH, 1975) e Brasil - RJ, MG, SP-( SOUZA \& SERRA-FREIRE, 1994 a,b; OLIVEIRA et al.,2000; LABRUNA et al.,2002), onde os picos de larvas desta espécie se concentraram na estação mais fria e seca, de abril a outubro. Essas diferenças estão possivelmente relacionadas ao fato de o presente estudo ter sido realizado em área de mata ciliar, onde foram coletadas duas espécies do gênero 


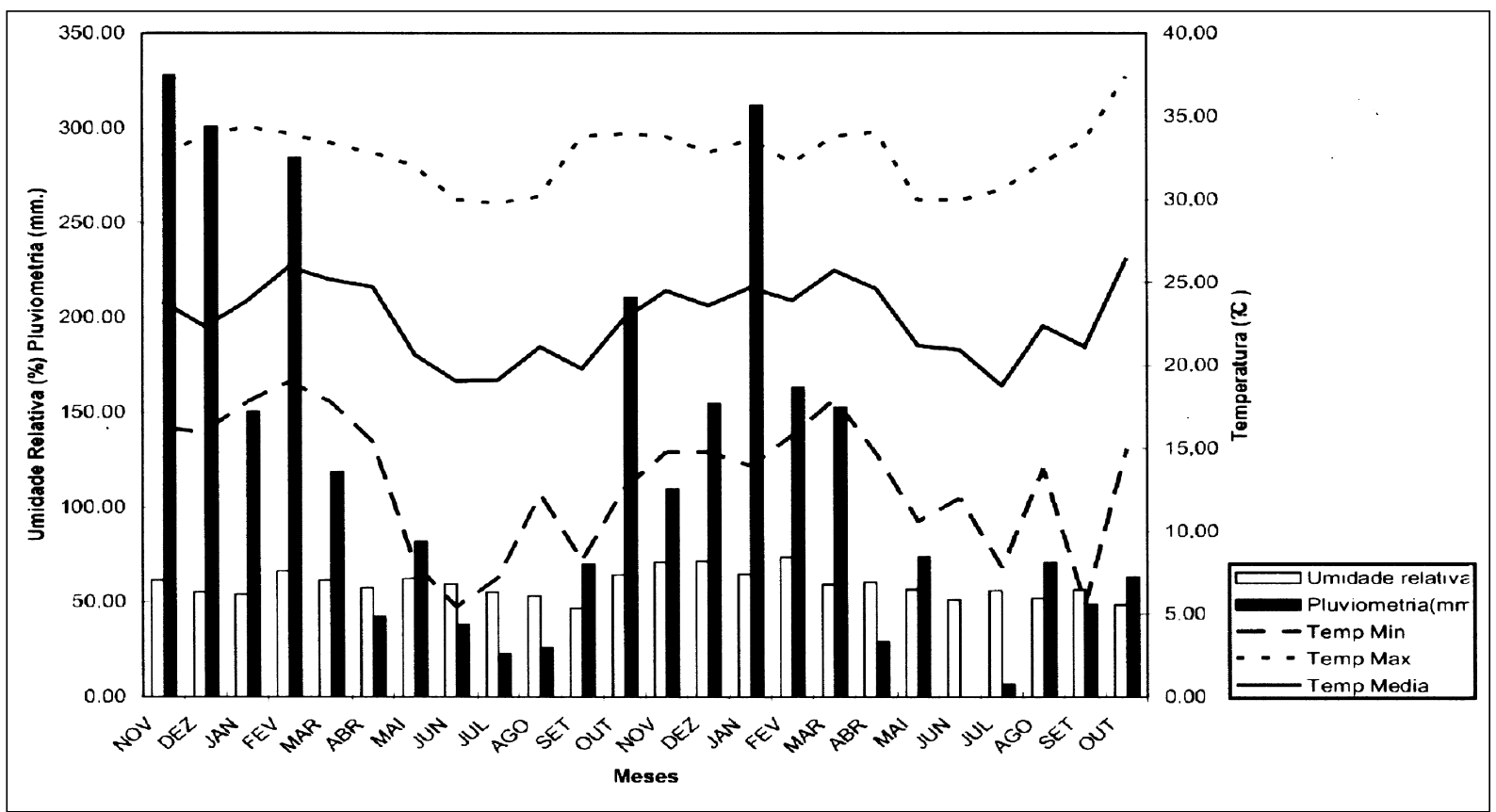

Figura 1 - Temperatura máxima, média e mínima, umidade relativa (\%) e pluviometria (mm). Novembro de 2000 a outubro de 2002. Campinas, SP.

Amblyomma (A. cajennense e A.dubitatum). Sendo assim, os picos de larvas ocorridos de abril a outubro possivelmente corresponderam, na sua maioria, a A.cajennense. Por outro lado, os picos de larvas ocorridos fora desse período (novembro a março), possivelmente corresponderam, na sua maioria, a larvas de A.dubitatum.

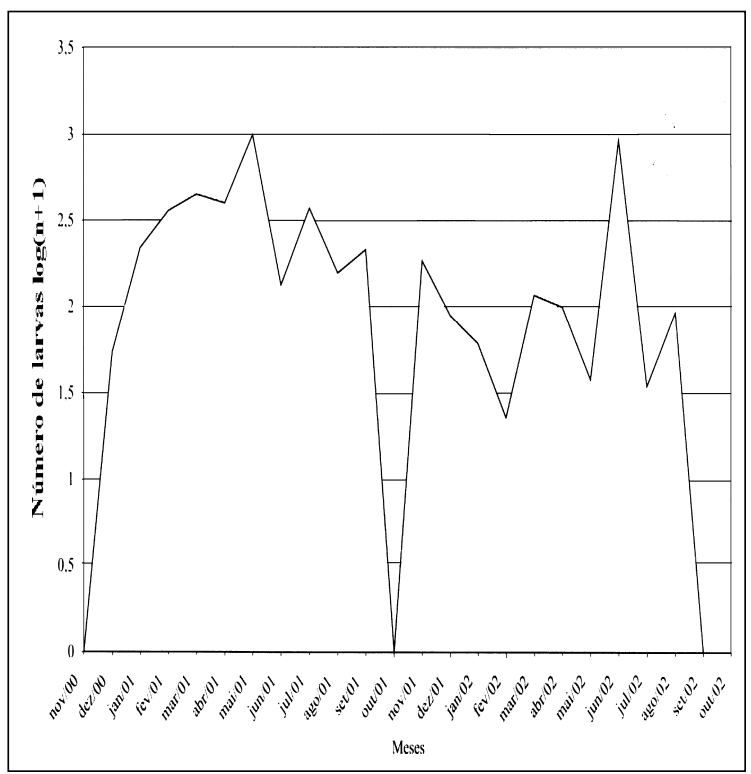

Figura 2 - Distribuição sazonal da popução de larvas de Amblyomma spp em ambiente de mata ciliar. Novembro de 2000 a outubro de 2002. Faz. Santa Júlia, Jaguariúna, SP.
O estádio de ninfa ocorreu o ano todo na mata ciliar, com maior abundância de julho dezembro, período esse que coincide com o aumento de casos de FMB na região estudada e em outras áreas endêmicas de SPe MG(LEMOS, 1996; GALVÃO, 1988). Os maiores picos de ninfas de Amblyomma spp observados no segundo semestre do ano possivelmente estão

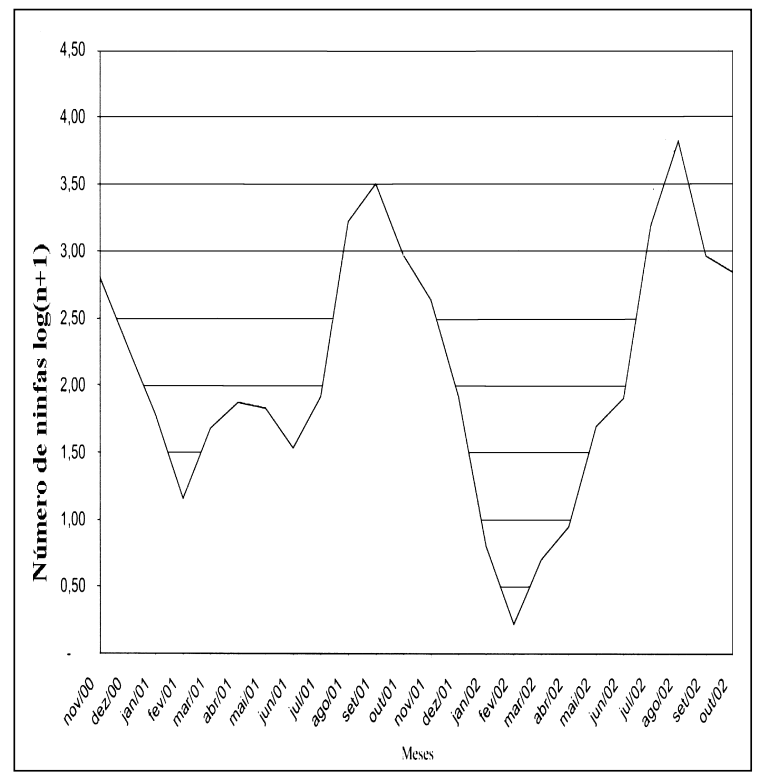

Figura 3 - Distribuição sazonal da população de ninfas de Amblyomma spp em ambiente de mata ciliar. Novembro de 2000 a outubro de 2002. Faz. Santa Júlia, Jaguariúna, SP.

Ciência Rural, v.36, n.3, mai-jun, 2006. 


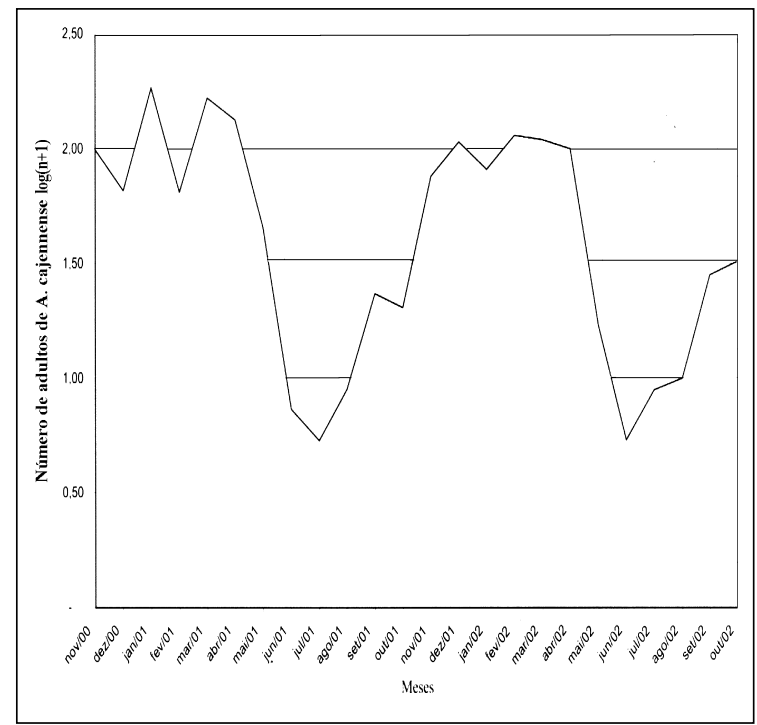

Figura 4 - Distribuição sazonal da população de adultos de Amblyomma cajennense em ambiente de mata ciliar. Novembro de 2000 a outubro de 2002. Faz. Santa Júlia, Jaguariúna, SP.

representados, na sua maioria, por ninfas de A.cajennense, uma vez que vários estudos no Brasil, Trinidad e Argentina mostraram que ninfas desta espécie ocorrem principalmente nesta época do ano, sendo raras no primeiro semestre do ano(SMITH, 1975; GUGLIELMONE \&HADANI, 1982; SOUZA \& SERRAFREIRE, 1994a,b; OLIVEIRA et al.,2000; LABRUNA et al., 2002). Em contrapartida, os picos de ninfas de Amblyomma spp observados no primeiro semestre do

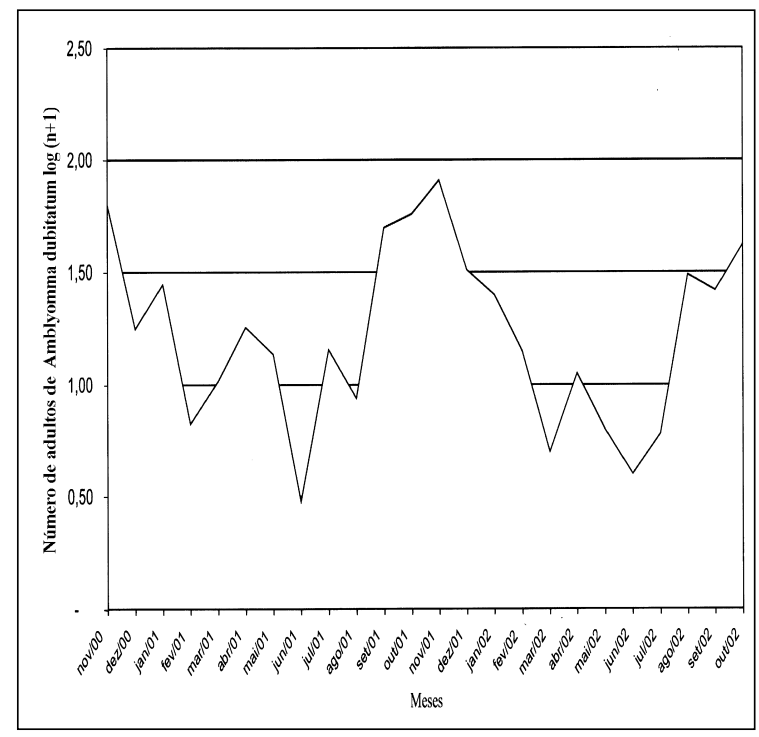

Figura 5 - Distribuição sazonal da população de adultos de Amblyomma dubitatum em ambiente de mata ciliar. Novembro de 2000 a outubro de 2002. Faz. Santa Júlia, Jaguariúna, SP. ano devem corresponder, na sua maioria, à espécie de A.dubitatum.

O aumento da população de adultos de A.cajennense na mata ciliar, de outubro a abril, coincidiu com os índices mais elevados de precipitação pluviométrica, umidade relativa e temperatura, entre novembro e março, estando de acordo com o padrão sazonal conhecido para esta espécie de carrapato na Região Sudeste do Brasil(SOUZA \& SERRA-FREIRE, 1994 a,b; LEMOS et al., 1997; OLIVEIRA et al.,2000; LABRUNA et al.,2002). Por outro lado, os adultos de A.dubitatum apresentaram os maiores picos a partir de julho, diferenciando-se do padrão observado para adultos de A.cajennense.

Alguns estudos demonstraram que o carrapato A.cajennense realiza apenas uma geração por ano na Região Sudeste do Brasil (SOUZA \& SERRAFREIRE, 1994 a,b; OLIVEIRA et al., 2000, LABRUNA et al., 2002), sendo este padrão controlado pela ocorrência de diapausa comportamental nas larvas não alimentadas, durante a primavera e o verão(LABRUNA et al., 2003). O presente estudo foi realizado em uma área de mata ciliar, onde duas espécies do gênero Amblyomma são freqüentes. Embora o padrão sazonal observado não permita concluir que a espécie A.cajennense esteja ocorrendo fora de seus padrões conhecidos para a Região Sudeste, foi constatado que o estádio adulto de A.dubitatum apresenta um padrão sazonal diferenciado. Futuros estudos deverão avaliar o padrão sazonal específico para larvas e ninfas de A.dubitatum, a fim de averiguar a ocorrência de diapausa e a duração de cada geração em condições naturais. Considerando a possibilidade de larvas e ninfas de A.dubitatum parasitarem humanos e de transmitirem o parasita Rickettsia parkeri (LABRUNA et al.,2004), causador de uma riquetsiose mais branda, a mata ciliar da Região de Campinas se mostra um local onde os humanos estariam expostos a carrapatos (e, conseqüentemente, a riquetsioses) por todo o ano.

\section{AGRADECIMENTOS}

À equipe de campo do Setor de São João da Boa Vista (Osmar, Laércio e Mário) e à diretora do SR5, Renata Caporalle Mayo, que colaboraram neste trabalho. Ao Marcelo Bahia Labruna, pelas sugestões referentes à discussão dos dados.

\section{REFERÊNCIAS}

ARAGÃO, H. Ixodidas brasileiros e de alguns paises limítrofes. Memórias do Instituto Oswaldo Cruz, Rio de Janeiro, v.31, n.3-4, p.759-843, 1936.

ARAGÃO, H.; FONSECA, F. Notas de Ixodologia. VIII. Lista e chave para os representantes da fauna ixodológica brasileira. 
Memórias do Instituto Oswaldo Cruz, Rio de Janeiro, v.59, n.2, p.115-148, 1961.

BURGDORFER, W. A review of Rocky Mountain spotted fever (tick-borne typhus), its agent, and its tick vectors in the United States. Journal Medical Entomology, Lanham, v.12, p.269278, 1975.

DIAS, E; MARTINS A.V. Spotted fever in Brazil. A summary. American Journal of Tropical Medicine and Hygiene,Baltimore, v.19, p.103-108, 1939

GALVÃO, M.A.M. A febre maculosa em Minas Gerais e seus determinantes. 1988. 186f. Dissertação ( Mestrado em Saúde Pública ) - Escola Nacional de Saúde Pública, Rio de Janeiro.

GUGLIELMONE, A.A. HADANI A. Amblyomma ticks found on cattle in northwest of Argentina. Annales de Parasitologie Humanie et comparee, Paris, v.57, p.91-97, 1982

GUIMARÃES, J.H. et al. Ácaros. In: Ectoparasitas de importância veterinária. São Paulo: Plêide, 2001. p.1-104.

JONES, E.K. et al. The ticks of Venezuela (Acarina: Ixodoidea) with a key to the species of Amblyomma in the Western Hemisphere. Brigham Young University Science Bulletin Biologycal, Provo, v.17, p.1-40, 1972.

LABRUNA, M.B. et al. Seasonal dynamics of ticks (Acari: Ixodidae) on horses in the state of São Paulo, Brasil. Veterinary Parasitology, Amsterdam, v.105, p.65-77, 2002.

LABRUNA, M.B. et al. Larval behavioral diapause regulates the life cycle of Amblyomma cajennense in Southeastern Brasil. Journal of Medical Entomology, Lanham, v.40, p.170-178, 2003.

LABRUNA, M.B. et. al. Rickettsia species infecting Amblyomma cooperi ticks from an area in state of São Paulo, Brazil, where Brazilian spotted fever is endemic. Journal of Clinical Microbiology, Washington, v.42, p.90-98, 2004

LEMOS, E.R.S. Febre maculosa brasileira em uma área endêmica no município de Pedreira, São Paulo, Brasil. 1996. 71f. Dissertação (Doutorado em Medicina Tropical) Instituto Oswaldo Cruz, Rio de Janeiro.

LEMOS, E.R.S. et al. Epidemiological aspects of the brazilian spotted fever: seasonal activity of ticks collected in an endemic area in São Paulo, Brazil. Revista da Sociedade Brasileira de Medicina Tropical, Uberaba, v.30, p.181-185, 1997.
LIMA, V.L.C. et al. Situação da febre maculosa na Região Administrativa de Campinas, São Paulo, Brasil. Cadernos de Saúde Pública, Rio de Janeiro, v.19, n.1, p.331-334, 2003.

MAGALHÃES, O. Contribuição para o conhecimento das doenças do grupo do tifo exantemático. Monog Instituto Oswaldo Cruz, Rio de Janeiro, n.6, 986f, 1952.

OLIVEIRA, P.R. et al. Population dynamics of the free-living stages of Amblyomma cajennense (Fabricius, 1787) (Acari: Ixodidae) on pastures of Pedro Leopoldo, Minas Gerais State, Brazil. Veterinary Parasitology, Amsterdan, v.92, p.295301, 2000

ROBINSON, L.E. The genus Amblyomma. Ticks, a monograph of the Ixodidae. Cambridge: Cambridge University, 1996. V.2, Part VI.

SMITH, M.W. Some aspects of the ecology and lifecycle of Amblyomma cajennense (Fabricius 1787) in Trinidad and their influence on tick control measures. Annals of Tropical Medicine and Parasitology, Liverpool, v.69, p.121-129, 1975.

SOLBERG, V.B. et al. Quantitative evaluation of sampling methods for Ixodes dammini and Amblyomma americanum (Acari: Ixodidae). Journal of Medical Entomology, Lanham v.29, p.451-456, 1992

SOUZA, A.P.; SERRA-FREIRE, N.M. Variação sazonal da fase não parasitária de Amblyomma cajennense e Boophilus microplus no município de Paracambi, Estado do Rio de Janeiro. Revista da Universidade Rural, Série Ciências da Vida, Rio de Janeiro, v.16, p.57-65, 1994a.

SOUZA, A.P.; SERRA-FREIRE, N.M. Variação sazonal da fase não parasitária de Amblyomma cajennense e Anocentor nitens no município de Itaguaí, Estado do Rio de Janeiro: Avaliação epidemiológica e metodológica. Revista da Universidade Rural, Série Ciências da Vida, Rio de Janeiro, v.16, p.6774, 1994b

TRAVASSOS, J.; VALLEJO FREIRE, A. Criação artificial de Amblyomma cajennense para o preparo da vacina contra a febre maculosa. Memórias do Instituto Butantan, Rio de Janeiro, v.18, p.145-235, 1944/1945.

WILSON, J.G et al. Chemo-attraction in the lone star tick (Acarina: Ixodidae). I. Response of different developmental stages to carbon dioxide administered via traps. Journal of Medical Entomology, Lanham, v.9, p.245-252, 1972. 